\title{
FACTORIZATION IN CODIMENSION ONE IDEALS OF GROUP ALGEBRAS
}

\author{
GEORGE WILLIS
}

\begin{abstract}
It is shown that if $G$ is a locally compact group and $I$ is a closed, two-sided ideal with codimension one in $L^{1}(G)$, then $I^{2}=I$.
\end{abstract}

Let $G$ denote a locally compact group, $L^{1}(G)$ be its group algebra with respect to left invariant Haar measure and $M(G)$ be the algebra of bounded Borel measures on $G$. As usual, we will identify $L^{1}(G)$ with the ideal of $M(G)$ consisting of measures which are absolutely continuous with respect to Haar measure. For each Banach algebra $A$, define

$$
A^{2}=\left\{\sum_{k=1}^{n} a_{k} b_{k} \mid a_{k}, b_{k} \in A, k=1, \ldots, n ; n \in \mathbf{N}\right\} .
$$

A question asked by B. E. Johnson in connection with certain automatic continuity problems (see [3, Example 6.3]) is whether $I^{2}=I$ when $I$ is a closed, two-sided, codimension one ideal in $L^{1}(G)$. This question may be answered immediately when $G$ is amenable, because in that case every codimension one ideal, $I$, in $L^{l}(G)$ has bounded approximate units (see [5]) and so, by Cohen's factorization theorem [1, 11.10], every element of $I$ is a product of two others. Here we answer Johnson's question in the nonamenable case.

THEOREM. Let $G$ be a locally compact group and I be a closed two-sided ideal with codimension one in $L^{1}(G)$. Then $I^{2}=I$.

Proof. Let $\chi_{I}$ be the continuous character on $G$ such that $I=\left\{f \in L^{1}(G) \mid\right.$ $\left.\int_{G} \chi_{I}(x) f(x) d x=0\right\}$ (see [4, 23.7]). Then the operator $T_{I}$, defined by $T_{I}(f)=\chi_{I} f$, is an automorphism of $L^{1}(G)$ and $T_{I}(I)=\left\{f \in L^{1}(G) \mid \int_{G} f(x) d x=0\right\}=I_{0}(G)$. Thus it suffices to prove the theorem in the case when $I=I_{0}(G)$. (This reduction was pointed out to me by B. E. Johnson.) Also, define $J_{0}(G)=\{\mu \in M(G) \mid \mu(G)=$ $0\}$.

Let $f$ be in $I_{0}(G)$. Then, since $L^{1}(G)$ has bounded approximate units [4, 20.27], it follows, by Cohen's factorization theorem, that there are elements $a$ and $b$ in $L^{1}(G)$ and $h$ in $I_{0}(G)$ such that $f=a * h * b$. We will show that

$$
h=\sum_{k=1}^{4} \mu_{k} * v_{k}, \quad\left(\mu_{k}, v_{k} \in J_{0}(G), k=1,2,3,4\right) .
$$

Received by the editors December 27, 1981.

1980 Mathematics Subject Classification. Primary 43A20; Secondary 46H10. 
The theorem will then follow because

$$
f=a * h * b=\sum_{k=1}^{4}\left(a * \mu_{k}\right) *\left(v_{k} * b\right),
$$

where $a * \mu_{k}$ and $v_{k} * b$ are in $I_{0}(G)$ for $k=1,2,3,4$.

Now let $r_{1}$ and $r_{2}$ be the real and imaginary parts of $h$, so that $r_{1}$ and $r_{2}$ are real valued functions on $G$ and $h=r_{1}+i r_{2}$. Then, since $h$ is in $I_{0}(G)$,

$$
\int_{G} r_{1}(x) d x=0=\int_{G} r_{2}(x) d x
$$

and $r_{1}$ and $r_{2}$ are also in $I_{0}(G)$. Define functions $r_{j}^{+}$and $r_{j}^{-}$for $j=1,2$ by

$$
\begin{aligned}
& r_{j}^{+}(x)= \begin{cases}r_{j}(x), & \text { if } r_{j}(x) \geqslant 0, \\
0, & \text { otherwise, }\end{cases} \\
& r_{j}^{-}(x)= \begin{cases}-r_{j}(x), & \text { if } r_{j}(x) \leqslant 0, \\
0, & \text { otherwise, }\end{cases}
\end{aligned}
$$

and define

$$
\lambda_{j}=\int_{G} r_{j}^{+}(x) d x \quad\left(=\int_{G} r_{j}^{-}(x) d x \text { because } r_{j}=r_{j}^{+}-r_{j}^{-}\right) .
$$

Finally, if $r_{j} \neq 0$ (so that $\lambda_{j}>0$ ), put $t_{j}^{ \pm}=r_{j}^{ \pm} / \lambda_{j}$.

With $t_{j}^{ \pm}$defined in this way we have that $t_{j}^{ \pm}(x) \geqslant 0$ for every $x$ in $G$ and $\int_{G} t_{j}^{ \pm}(x) d x=1$, for $j=1,2$. Hence,

$$
h=\lambda_{1}\left(e-t_{1}^{-}\right)-\lambda_{1}\left(e-t_{1}^{+}\right)+i \lambda_{2}\left(e-t_{2}^{-}\right)-i \lambda_{2}\left(e-t_{2}^{+}\right),
$$

where $\left\|t_{j}^{ \pm}\right\|=1$ and $e$ is the identity element in $M(G)$, so that $e-t_{j}^{ \pm}$is in $J_{0}(G)$ for $j=1,2$.

The Theorem now follows because, if $t$ in $L^{1}(G)$ is such that $t(x) \geqslant 0$ for every $x$ in $G$ and $\|t\|=1$, then $e-t$ has a square root in $J_{0}(G)$ defined by the binomial expansion

$$
(e-t)^{1 / 2}=\sum_{n=0}^{\infty}\left(\begin{array}{c}
1 / 2 \\
n
\end{array}\right)(-t)^{n}
$$

The series converges because the coefficients $\left\{\left(\begin{array}{c}1 / 2 \\ n\end{array}\right)\right\}_{n=0}^{\infty}$ form an $l^{1}$-sequence and $\left\|t^{n}\right\|=1$ for every $n$. That $(e-t)^{1 / 2}$ is in $J_{0}(G)$ follows because $e-t$ is and because $J_{0}(G)$ is the kernel of a multiplicative linear functional on $M(G)$.

The last part of the proof in fact shows the following result for $J_{0}(G)$.

COROLlaRY. Let $G$ be a locally compact group. Then $J_{0}(G)^{2}=J_{0}(G)$.

However, in spite of this, the theorem does not hold if $L^{1}(G)$ is replaced by $M(G)$. It is shown in [2] that, if $G$ is a nondiscrete abelian group, then there is a codimension one, closed ideal $I$ in $M(G)$ such that $I^{2} \neq I$.

The work in this paper formed part of my doctoral thesis completed at the University of Newcastle-upon-Tyne. I am grateful to Professor B. E. Johnson for his helpful supervision. 


\section{REFERENCES}

1. F. F. Bonsall and J. Duncan, Complete normed algebras, Springer-Verlag, Berlin and New York, 1973.

2. G. Brown and W. Moran, Point derivations on $M(G)$, Bull. London Math. Soc. 8 (1976), 57-64.

3. H. G. Dales, Automatic continuity: a survey, Bull. London Math. Soc. 10 (1978), 129-183.

4. E. Hewitt and K. A. Ross, Abstract harmonic analysis, Vol. I, Springer-Verlag, Berlin and New York, 1963.

5. H. Reiter, Sur certains idéaux dans $L^{1}(G)$, C. R. Acad. Sci. Paris Sér. A-B 267 (1968), 882-885.

Department of Mathematics, University of New South Wales, Kensington, New South Wales, Australia 2033 